\title{
Pattern Dependency of Pure-Boron-Layer Chemical-Vapor Depositions
}

\author{
V. Mohammadi, W.B. de Boer, T.L.M. Scholtes, L.K. Nanver \\ Delft Institute of Microsystems and Nanoelectronics (Dimes), \\ Delft University of Technology, \\ Feldmannweg 17, 2628 CT Delft, The Netherlands, \\ Phone: +31 (0)15 27 86294, Fax: +31 (0)15 2787369 , \\ E-mail: v.mohammadi@tudelft.nl
}

\begin{abstract}
The pattern dependency of pure-boron (PureB) layer chemicalvapor depositions (CVD) is studied with respect to the correlation between the deposition rate and features like loading effects, deposition parameters and deposition window sizes. It is shown experimentally that the oxide coverage ratio and the size of windows to the $\mathrm{Si}$ on patterned wafers are the main parameters affecting the deposition rate. This is correlated to the gas depletion of the reactant species in the stationary/low-velocity boundary layer over the wafer. An estimation of the radius of gas depletion for Si openings and/or diffusion length of diborane in this study yields lengths in the order of centimeters, which is related to the boundary layer thickness. The deposition parameters; pressure and flow rates are optimized to minimize the pattern dependency of the PureB deposition rates.
\end{abstract}

\section{Introduction}

The chemical-vapor deposition of pure boron has in the last years been very successfully applied for fabricating extremely shallow, less than 10-nm deep, silicon $p^{+} n$ junction diodes for a number of leading-edge device applications. This so-called PureB technology has provided particularly impressive performance of photodiode detectors for low penetration-depth beams for which 2-nm-thick PureB-layers are reliably implemented as the front-entrance window (1-4). Ideal low-leakage diode characteristics are achieved for deposition temperatures from $400^{\circ} \mathrm{C}-700^{\circ} \mathrm{C}$, which together with the fact that the deposition is conformal and highly selective to $\mathrm{Si}$, could also make PureB technology an attractive candidate for creating junctions on silicon nanowires and advanced CMOS transistors including source/drain in p-type FinFETs (5-7). In the latter applications, sub-3-nm thick layers are required to avoid excess series resistance through the high-resistivity boron layer. Therefore, a very good control of the layer thickness is crucial, in which respect the uniformity over the wafer and the pattern dependence are the important factors.

In this paper the pattern dependency of the PureB deposition is studied for different sizes and distributions of deposition windows etched in oxide to the Si. Loading effects are observed that in some ways similar to those found for selective epitaxial growth (SEG) of Si and SiGe layers. Differences in the deposition rate of the deposited layers are correlated to the $\mathrm{Si} / \mathrm{SiO}_{2}$ ratio over the wafer as a global effect and the size of the windows in relationship to the pattern of the surrounding oxide areas as a local effect. 
This pattern dependency must be taken into account when applying the PureB deposition to devices where the boron-layer thickness is critical as, for example, in photodiodes for detecting electrons with energies below about $1 \mathrm{keV}$ (1). Significant non-uniformities can be found when window sizes are in the range of millimeters or centimeters.

In this paper we focus on determining the global effects that can be determined on large area windows by using measurement techniques such as SIMS or ellipsometry. While several methods have been proposed to decrease the pattern dependency and/or loading effect for SEG of the $\mathrm{Si}$ and $\mathrm{SiGe}(8-10)$, this is the first report on the reproducibility and the pattern dependency including loading effects of the PureB depositions. An optimization of the deposition parameters is performed for $700^{\circ} \mathrm{C}$ depositions, at either atmospheric (ATM) pressure or 60 torr, with respect to uniformity over the wafer with and without a patterned oxide coverage. As a result deposition procedures are proposed for achieving a well-control deposition rate with very low pattern dependency.

\section{Experimental Procedure}

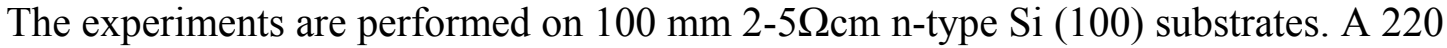
$\mathrm{nm}$-thick thermal oxide is grown on some wafers and patterned by wet etching in BHF. The PureB deposition is performed by an atmospheric/low pressure-chemical vapor deposition (AP/LP-CVD) at $700{ }^{\circ} \mathrm{C}$ in an ASM Epsilon $2000 \mathrm{CVD}$ reactor. Diborane is used as a gas source and hydrogen as both carrier gas and dilutant (6). Both bare and patterned wafers were ex-situ standard cleaned $\left(\mathrm{HNO}_{3} 100 \%\right.$ and $\mathrm{HNO}_{3} 70 \%$ at $110^{\circ} \mathrm{C}$ and HF dip with Marangoni drying) and then immediately loaded to the $\mathrm{N}_{2}$ purged load locks of the reactor. In-situ cleaning was performed for bare and patterned wafers by baking in $\mathrm{H}_{2}$ prior to the deposition process at $1100^{\circ} \mathrm{C}$ and $800^{\circ} \mathrm{C}$ for 2 and $4 \mathrm{~min}$, respectively.

Eight samples in four groups with different pattern sizes and different oxide coverage ratio (OCR) were used in this study to investigate pattern dependency, as presented in Table 1. An oxide coverage ratio can be defined here as the ratio of the oxide masked area to the entire surface area of the wafer:

$$
\operatorname{OCR}(\%)=\frac{S_{\mathrm{SiO}_{2}}}{S_{\text {Wafer }}} \times 100
$$

TABLE 1. Description of Samples

\begin{tabular}{|l|c|c|l|}
\hline Sample & OCR (\%) & Window areas $\mathbf{( m m}^{\mathbf{2}} \mathbf{)}$ & Comment \\
\hline SW-58 & 58 & 43.7 & and many small windows \\
\hline SW-71 & 71 & 7.68 & and many small windows \\
\hline 6LW-80 & 80 & $50,75,100,200,300,400$ & 6 window sizes \\
\hline 4LW-80 & 80 & $100,200,300,400$ & large windows of different sizes \\
\hline 14LW-80 & 80 & 100 & Fourteen windows \\
\hline LW-SiOpen & 71 & 100,81 & 27 dies \\
\hline LW-Oxide & 72 & 81 & 27 dies \\
\hline LW-SiDies & 75 & 81 & 24 dies \\
\hline
\end{tabular}


Ellipsometry measurements were used in order to measure the thickness of the PureBlayer at the middle of millimeter to centimeter large windows to the $\mathrm{Si}$. For wafers without any oxide coverage the measurement is performed in the middle of the wafer.

\section{Results and Discussion}

The influence of the deposition parameters (e.g., pressure, temperature and gas flow) and structural parameters (e.g., aperture size, oxide coverage ratio) on the pattern dependency and/or loading effects of the PureB-layers is a complicated issue since the parameters can be interdependent. Ideally, a deposition should be totally independent of the size/geometry of the openings. However, in reality, many effects play a role due to the non-uniform gas consumption over different window sizes and different lateral gas diffusion because of global/local loading effects. The exact origin of the pattern dependency is a complex combination of chemical, kinetical, and thermal factors, which are evaluated here in a systematic experimental study.

\section{Effect of oxide coverage and window size}

In order to investigate the effect of $\mathrm{Si}$ opening size and oxide coverage on the wafer defined here as an OCR, the deposition rate is compared for samples SW-58, SW-71 and 6LW-80. In Fig. 1 the dependency is plotted as a function of OCR for wafers with different patterns. As the oxide coverage ratio goes from 0 to $80 \%$, the deposition rate increases to about $85 \%$. Also plotted in Fig. 1 is the deposition rate as a function of the window size for the same OCR. All the measurements are performed on wafer 6LW-80, which means that there is no influence from global effects. Due to a loading effect the deposition rate is increased as the feature size of the Si windows decreases. These results demonstrate the general trend that the deposition rate will increase as the area covered by oxide increases either globally and in the direct vicinity of an opening to Si.

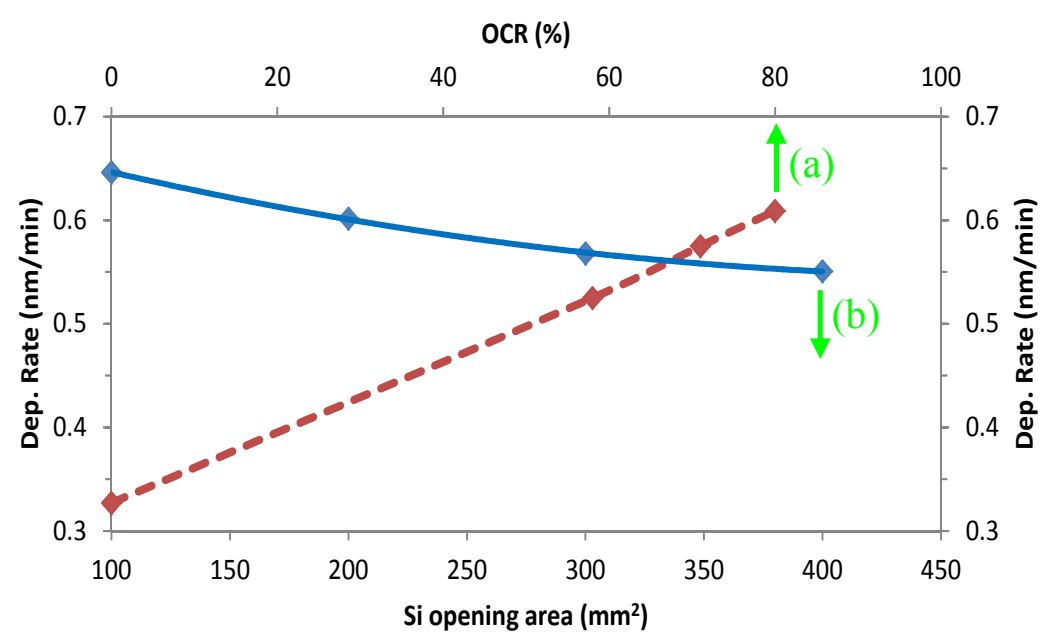

Figure 1. The deposition rate of the PureB-layer as a function of (a) oxide coverage ratio (dashed line) for a wafer with no oxide and samples SW-58, SW-71 and 6LW-80, and (b) the area of the individual oxide windows to $\mathrm{Si}$ on sample $6 \mathrm{LW}-80$, i.e., with a constant OCR of $80 \%$ (solid line). 
These trends are related to the selective nature of the deposition and are also wellknown from selective silicon epitaxy $(12,13)$. CVD deposition on blanket wafers may be described using the classical boundary layer theory (14). In this theory, a laminar gas stream is flowing over the wafer. Due to the frictional force between the gas stream and the stationary susceptor/substrate, a stagnant/low-velocity boundary-layer arises. Therefore the main gas transport mechanism through this boundary layer is diffusion. With the reaction of the reactant species with the surface atoms and subsequent deposition, the boundary layer will become depleted if the net deposition reaction is faster than the diffusion. Over this boundary layer, the gas is assumed to be well mixed and moving at a constant speed $U$. To determine the thickness of this boundary layer, the balance of the frictional and accelerating forces on a gas element yields a boundary layer thickness $\delta$ as a function of the position over the susceptor/substrate, $x$, to be $\delta(x)=A(\mu x / U)^{1 / 2}$, where $\mu$ is the gas kinematical viscosity and $A$ is a constant. However, the thickness of this boundary layer is not easy to calculate exactly. Several unknown parameters are involved, for example, kinematical viscosity and gas velocity are highly dependent on the actual gas temperature which can be significantly different from the susceptor temperature. This is typical for lamp-heated cold-wall reactors, where the temperature is controlled by thermocouples located in the susceptor. A rough approximation of the magnitude of this boundary layer yields values in the centimeter range. This means that the reactant species have to diffuse vertically in the range of centimeters. However, diffusion will also occur laterally if there are any concentration gradients over the wafer (which is the case for selective deposition on substrates with varying window sizes). This implies that the gas phase depletion gradient may be significant (in the centimeter range) both vertically and laterally.

In the case of PureB depositions, it has been found that no boron is deposited on the flat or beveled $\mathrm{SiO}_{2}$ surfaces such as confirmed by the TEM image in Fig. 2.

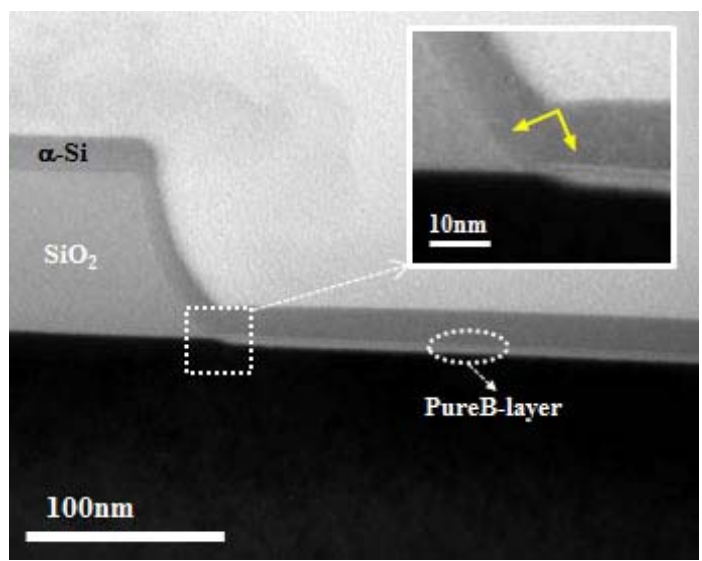

Figure 2. TEM image of a B-layer directly formed in a contact window during a 6 min $\mathrm{B}_{2} \mathrm{H}_{6}$ exposure at $700^{\circ} \mathrm{C}$.

Therefore, the boron atoms on the oxide are floating and move around on the surface thus creating higher concentration boron atoms that will give a higher lateral diffusion on a patterned wafer surface than on a bare-Si wafer. Consequently there is an increase of the probability of the boron atoms sticking to the Si surface of the open windows. In this way an increase of the amount of oxide area around the window will result in an increase in the PureB-layer thickness. 
$\underline{\text { Impact of deposition parameters on pattern dependency }}$

A series of reports on SEG of Si and SiGe, have shown that the pattern dependency can be reduced by tuning the growth parameters (8-10). Here the PureB-layer was deposited at both atmospheric pressure (ATM) and 60 torr on several patterned wafers with $\sim 80 \%$ OCR and opening areas of $100 \mathrm{~mm}^{2}, 200 \mathrm{~mm}^{2}, 300 \mathrm{~mm}^{2}$ and $400 \mathrm{~mm}^{2}$ at atmospheric pressure (ATM) and 60 torr. Deposition was performed with different diborane partial pressures and main gas flows which $\mathrm{P}_{1}\left(\mathrm{~F}_{1}\right)$ is the maximum diborane partial pressure (main flow) and $\mathrm{P}_{2}\left(\mathrm{~F}_{2}\right)$ and $\mathrm{P}_{3}\left(\mathrm{~F}_{3}\right)$ are $75 \%$ and $50 \%$ of the maximum values. The PureB deposition rate is shown in Fig. 3 as a function of these parameters. In each figure the corresponding deposition rate for bare wafers is also shown. The results show a reduction of the pattern dependency with decreasing diborane partial pressure as well as main gas flow (Fig. 3a and 3b). Moreover, no significant improvement in the pattern dependency was observed when decreasing the total pressure from ATM to 60 torr, see Fig 3c.
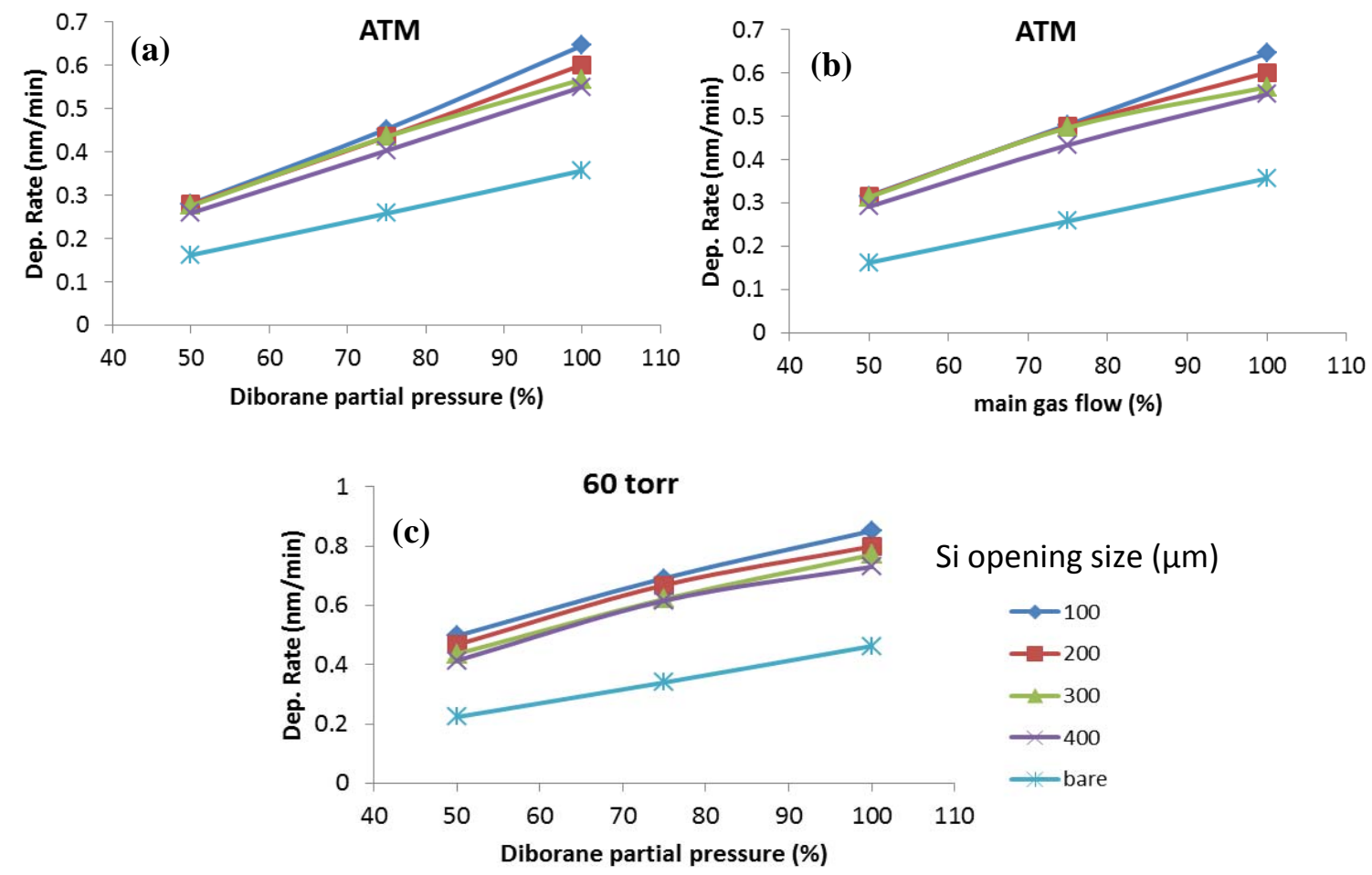

Figure 3. The PureB deposition rate as a function of (a) diborane partial pressure (b) main gas flow at atmospheric pressure and (c) diborane partial pressure at 60 torr, for different window sizes as well as for a bare Si wafer.

In order to verify the effect of the deposition parameters and surrounded oxide areas on the pattern dependency and/or deposition rate of the PureB, the pattern which is shown in Fig. 4 (samples 14LW-80) was designed. The PureB was deposited over this design where 14 oxide windows to the silicon, each $1 \times 1 \mathrm{~cm}^{2}$ in size, are positioned so that the surrounding oxide area is different in each case. This gives different loading effects and therefore different PureB deposition rates. 


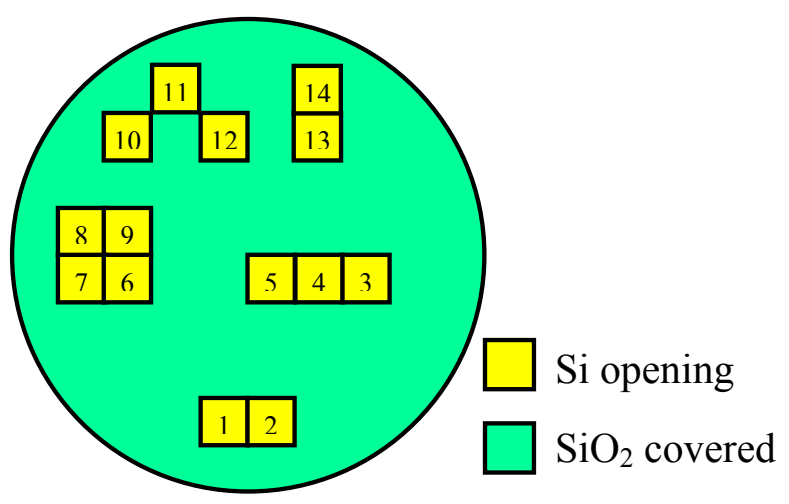

Fig. 4. Patterning of the samples 14LW-80.

In Fig. 5 the results are shown for different diborane partial pressure and main gas flow combinations. As can be seen in this figure, e.g., dies no. 3 and 5 have a higher deposition rate due to the largest amount of adjacent oxide areas and die no. 11 has a lower value because it is located near two another dies and close to the edge of the wafer with less surrounded oxide areas. The large variations become very small when the diborane partial pressure and main gas flow are low. However, as seen in Fig 5b, the deposition rate variations are not significantly changed by reducing the total pressure from ATM to 60 torr.
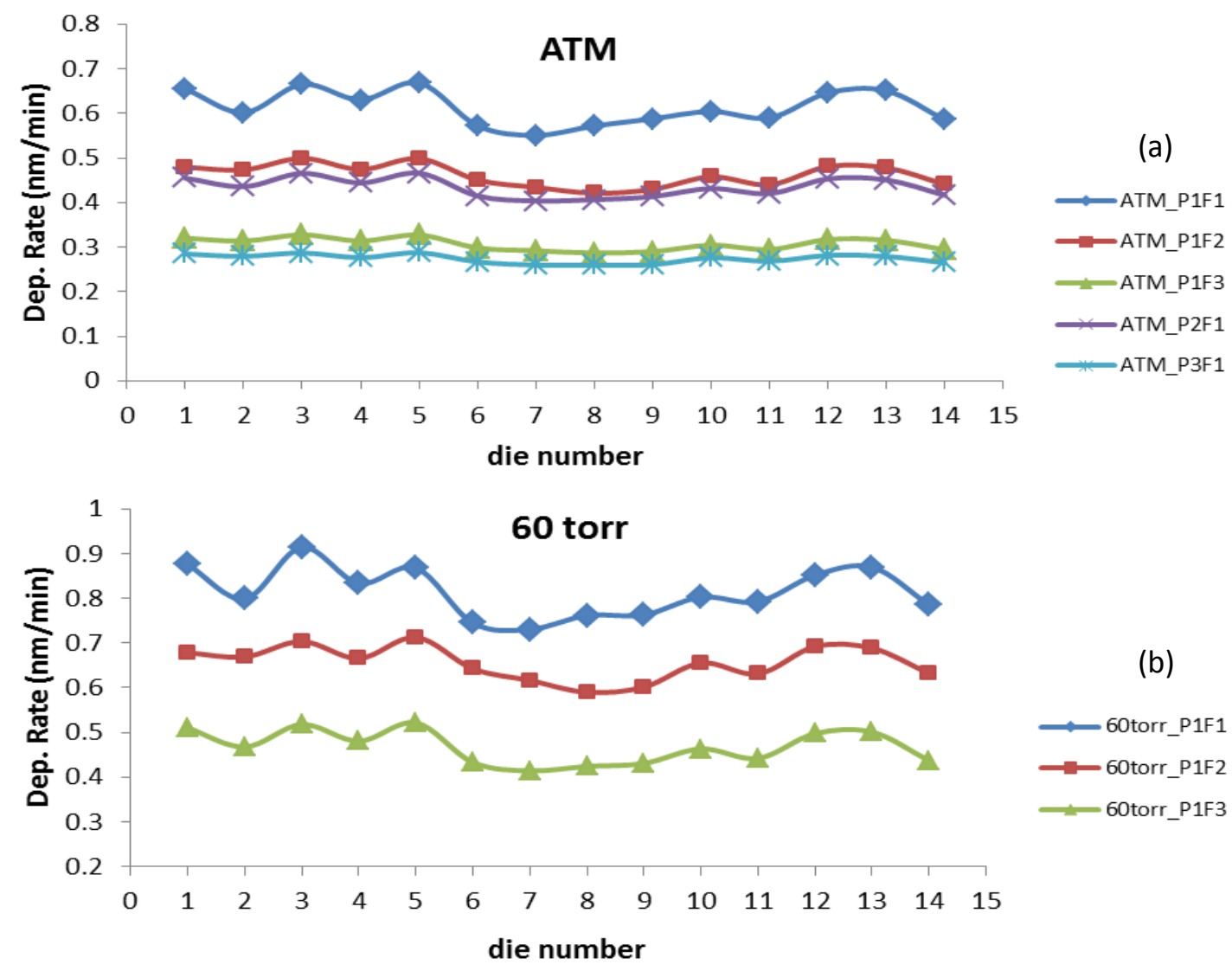

Figure 5. The PureB deposition rate plotted against the die number for a wafer patterned with $141 \times 1 \mathrm{~cm}^{2}$ dies as in the wafer layout of Fig. 4, for different deposition parameters at (a) atmospheric pressure, (b) 60 torr pressure. Here $\mathrm{P}_{1}\left(\mathrm{~F}_{1}\right)$ is the maximum diborane partial pressure (main gas flow) and $\mathrm{P}_{2}\left(\mathrm{~F}_{2}\right)$ and $\mathrm{P}_{3}\left(\mathrm{~F}_{3}\right)$ are $75 \%$ and $50 \%$ of the maximum values, respectively. 
These results show that for atmospheric pressure, the deposition rate for maximum diborane partial pressure and maximum main flow $\left(\mathrm{P}_{1} \mathrm{~F}_{1}\right)$ varies the most due to a high pattern dependency and/or loading effect from the surrounding oxide areas. However with decreasing diborane partial pressure or main flow (e.g. $\mathrm{P}_{1} \mathrm{~F}_{2}, \mathrm{P}_{1} \mathrm{~F}_{3}, \mathrm{P}_{2} \mathrm{~F}_{1}$, and $\mathrm{P}_{3} \mathrm{~F}_{1}$ ) this effect becomes much lower and all dies with any adjacent oxide areas and also with different width (e.g. dies no. 6, 7, 8 and 9 together) have the same deposition rate. At the same time total deposition rate is decreased as would be expected. This experiment shows that it is possible to adjust the deposition parameters so that the loading effect of the oxide areas can be regulated to give a uniform and equally thick PureB-layer in all the dies of a patterned wafer.

\section{Verification of the gas depletion in the boundary layer}

As discussed previously, windows to the Si consume the diborane species and cause gas depletion inside the boundary layer over the windows. Therefore the diborane species have to diffuse through the stationary boundary layer width from the main gas stream to reach the surface of the wafer which is schematically illustrated in Fig. 6. Again, the diborane atoms cannot stick onto the oxide areas and boron concentration is increased over these areas. Therefore, also the lateral gas diffusion is increased, which creates a local boundary layer over each opening as is shown by the yellow arrows in the Fig. 6 .

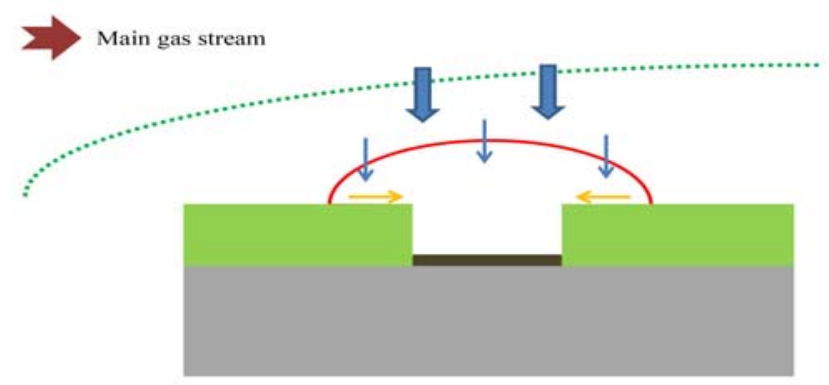

Figure 6. Schematic illustration of local boundary layer over a window to Si due to the lateral diffusion of the boron species.

Generally, many different windows with different distances from each other are found on each die or wafer, and the deposition rate will also be influenced by the proximity of neighboring windows. This can be put in relationship to the width of the opening windows, $\mathrm{d}_{\mathrm{Si}}$, and width of the oxide, $\mathrm{d}_{\mathrm{ox}}$, separating the windows. This situation is shown schematically in Fig. 7.

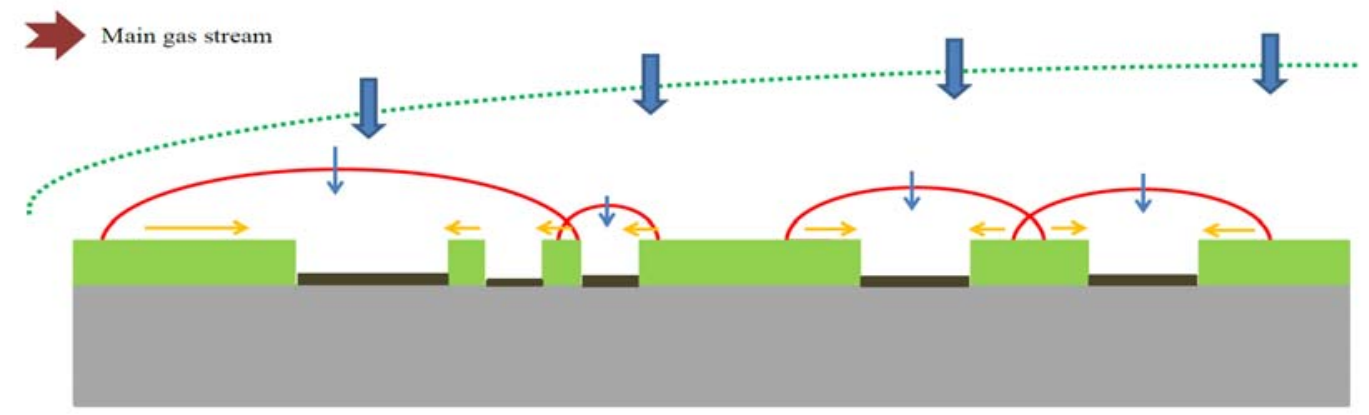

Figure 7. Schematic illustration of local boundary layers and their impact on the final PureB layer-thickness for a die design where equal windows or oxide separation sizes result in different thicknesses. 
To verify the boundary layer thickness and range of gas depletion in the boundary layer, PureB was deposited on wafers patterned as shown in Fig. 8. Three wafers were processed with the area designated as the "reference" area filled in different ways:

LW-SiOpen: one big window to the $\mathrm{Si}$,

$L W$-Oxide: covered by oxide,

LW-SiDies: three $10 \times 10 \mathrm{~mm}^{2}$ dies are opened as separated windows to the $\mathrm{Si}$.

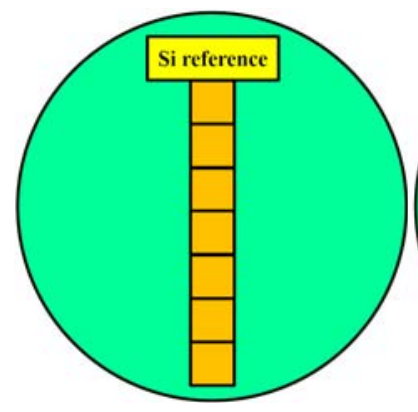

LW-SiOpen

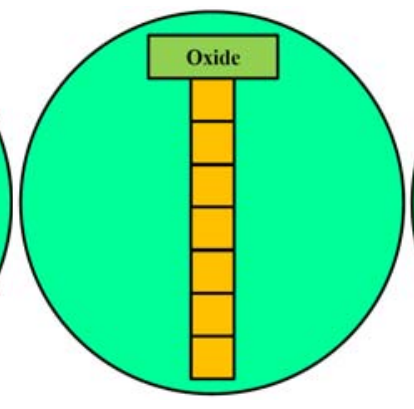

LW-Oxide

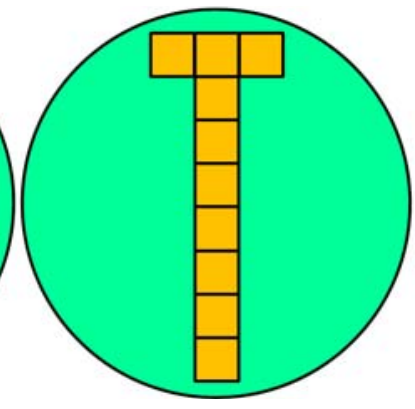

LW-SiDies

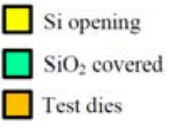

Figure 8. Patterning of wafers where the "reference" area is filled in different ways for samples LW-SiOpen, LW-Oxide and LW-SiDies.

If the dominant transport mechanism is diffusion and the boundary layer thickness is of the order of centimeters, then the extent of gas depletion from a big opening should also be in the order of centimeters. Figure 9 shows the deposition rate of the PureB in the $10 \times 10 \mathrm{~mm}^{2} \mathrm{Si}$ openings at different distances from the "reference" area for the three samples.

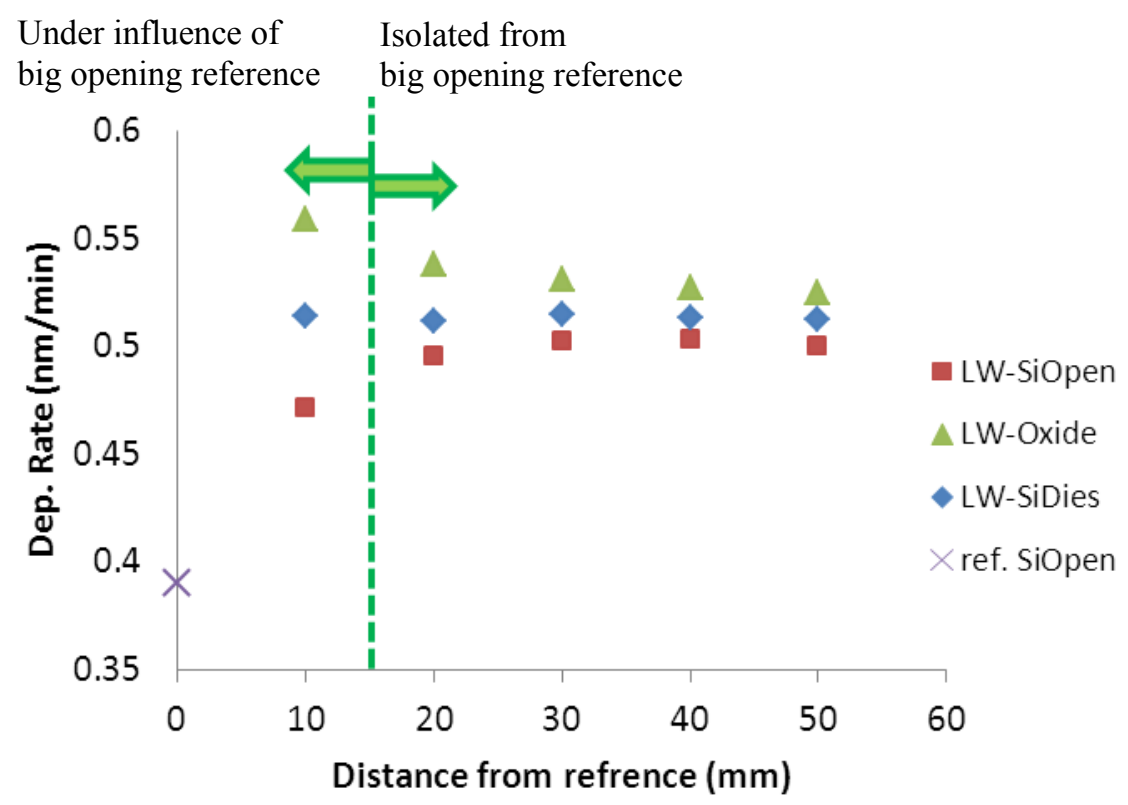

Figure 9. The deposition rate of the PureB in a row of five $1 \mathrm{x} 1 \mathrm{~cm}^{2}$ test dies adjacent to a $3 \times 1 \mathrm{~cm}^{2}$ die placed as the "reference" area shown in Fig. 8 for three different fillings of this area as defined for the sample LW-SiOpen, LW-Oxide and LW-SiDies. The deposition rate in the "Si reference" area for sample LW-SiOpen is included for comparison. 
For comparison, the PureB deposition rate in the "reference" area for sample LWSiOpen is included. The curve for design LW-SiOpen reveals two distinct regions. Close to the reference area, the smaller openings have a low deposition rate, lower than that of the dies that are further away and not impacted by the depletion volume of the large reference opening as schematically indicated in Fig. 7. In this volume, a significant part of the gas molecules is consumed by this large opening and the diborane available for the surrounding dies is reduced. As the distance is increased, the deposition rate is also increased until saturation is reached at a distance of around $1-1.5 \mathrm{~cm}$.

The curve for design LW-Oxide has also two distinct regions. In this design the "reference" is covered by oxide. The loading effect of this oxide area increases the deposition rate of the closest open dies. It can be also deduced that the diffusion length of diborane on the oxide area should be around 1-1.5 cm. From Fig. 9, it can be deduced that when the "reference" area is patterned with separated open dies of the same area as the five dies forming the monitoring row then the dies do not have a distinguishable impact on each other. All in all, it can be concluded that considering the fact that the smaller openings display the same deposition behavior as the nearby larger opening means that any micro-loading effects are minimal, i.e., there is no dependence on the opening size but the local Si coverage is very important. It should be noted that the uniformity of the deposition over a blanket wafer under these deposition conditions was checked and the nonuniformity was less than $2 \%$. Therefore the distribution of PureBlayer thickness over these test wafers supports the picture of the deposition and associated of pattern dependency of PureB CVD as illustrated by Fig. 7.

\section{Conclusions}

It has been demonstrated that the deposition rate of PureB can vary over the wafer depending on the Si opening size and OCR of the mask design. The higher the OCR the higher the deposition rate and it also increases as the sizes of the windows to the $\mathrm{Si}$ decrease. This has been explained here in terms of the gas depletion of the diborane species in the stationary and/or local boundary layer. The results show a reduction of the pattern dependency with decreasing diborane partial pressure as well as main gas flow. Moreover, no significant improvement in the pattern dependency was observed when decreasing the total pressure from ATM to 60 torr. Thus conditions were found that make it possible to control pattern dependency and loading effects to such a degree that the 2nm-thick PureB-layers used in the present day applications can be deposited uniformly with only a few angstrom variation in thickness. The diffusion lengths of the boron on the oxide and Si surfaces is found to be in the order of centimeters, which means that the loading effect in micron sized windows will not lead to thicker layers than seen in the larger windows.

\section{Acknowledgements}

The authors would like to thank the staff of the DIMES-ICP cleanrooms for their support in the fabrication and measurement of the experimental material. This work was supported by Project No. 10024 of The Dutch Technology Foundation STW. 


\section{References}

1. A. Šakić, L.K. Nanver, G. van Veen, K. Kooijman, P. Vogelsang, T. L.M. Scholtes, W. de Boer, W.H.A. Wien, S. Milosavljević, C.Th.H. Heerkens, T. Knežević and I. Spee, "Versatile Silicon Photodiode Detector Technology for Scanning Electron Microscopy with High-Efficiency Sub-5 keV Electron Detection," IEDM'10, pp.712-713, 2010.

2. L. Shi, F. Sarubbi, L.K. Nanver, U. Kroth, A. Gottwald and S. Nihtianov, "Optical performance of B-layer ultrashallow-junction Silicon photodiodes in the VUV spectral range," Proc. Eurosensors XXIV, pp. 633-636, 2010.

3. L. Shi, F. Sarubbi, S.N. Nihtianov, L.K. Nanver, T.L.M. Scholtes, F. Scholze, "High performance silicon-based extreme ultraviolet (EUV) radiation detector for industrial application," 35th Annual Conference of IEEE Industrial Electronics Society (IECON), 2009, pp. 1877-1882.

4. L.K. Nanver, "Silicon Photodiodes for Low Penetration Depth Beams such as DUV/VUV/EUV Light and Low-Energy Electrons", Advances in Photodiodes, Book edited by: Gian Franco Dalla Betta, ISBN: 978-953-307-163-3, Publisher: InTech, Publishing date: March 2011.

5. L.K. Nanver, T.L.M. Scholtes, F. Sarubbi, W.B. de Boer, G. Lorito, A. Šakić, S. Milosavljević, C. Mok, L. Shi, S. Nihtianov, K. Buisman, "Pure-Boron ChemicalVapor-Deposited Layers: a New Material for Silicon Device Processing," 18th IEEE Conference on Advanced Thermal Processing of Semiconductors (RTP), pp. 136-139, 2010.

6. F. Sarubbi, T.L.M. Scholtes, and L.K. Nanver, "Chemical Vapor Deposition of $a$ Boron Layers on Silicon for Controlled Nanometer-Deep $\mathrm{p}^{+} \mathrm{n}$ Junction Formation," J. of Electron. Mater., Vol. 39 (2), pp. 162-173, February 2010.

7. F. Sarubbi, L.K. Nanver, and T.L.M. Scholtes, "High effective Gummel number of CVD boron layers in ultrashallow $\mathrm{p}^{+} \mathrm{n}$ diode configurations," IEEE Trans. Electron Devices, vol. 57 (6), pp. 1269-1278, 2010.

8. M. Kolahdouz, P. Tabib Zadeh Adibi, A. Afshar Farniya, S. Shayestehaminzadeh, E. Trybom, L. Di Benedetto, and H.H. Radamson, "Selective Growth of B- and C-Doped SiGe Layers in Unprocessed and Recessed Si Openings for p-type Metal-Oxide-Semiconductor Field-Effect Transistors Application," Journal of The Electrochemical Society, 157 (6), pp. H633-H637, 2010.

9. M. Kolahdouz, J. Hållstedt, M. Ostling, R. Wise and H.H. Radamson, "Selective Epitaxial Growth with Full Control of Pattern Dependency Behavior for pMOSFET Structures,” J. Electrochem. Soc., 156 (3), pp. H169-H171, 2009.

10. C. Menon, A. Bentzen, and H.H. Radamson, "Loading effect in SiGe layers grown by dichlorosilaneand silane-based epitaxy," J. Appl. Phys., 90 (9), 2001.

11. A. Šakić , T.L.M. Scholtes, W. de Boer, N. Golshani, J. Derakhshandeh and L.K. Nanver, "Arsenic-Doped High-Resistivity-Silicon Epitaxial Layers for Integrating Low-Capacitance Diodes," Materials 2011, 4(12), 2092-2107.

12. A. Ishitani, N. Endo and H. Tsuya, "Local Loading Effect in Selective Silicon Epitaxy," Jap. J. of Applied Physics, vol.23 (6), pp. L391-L393, 1984.

13. R. Pagliaro, J.F. Corboy, L.Jastrzebski and R.Soydan, "Uniformly Thick Selective Epitaxial Silicon", J. Electrochem. Soc., vol. 134 (5), p. 1235, 1987.

14. J.D. Plummer, M. Deal, P.D. Griffin (Author), Silicon VLSI Technology: Fundamentals, Practice, and Modeling, ch.9, Prentice Hall (2000). 